\title{
Solving the Transmembrane Potential Based Inverse Problem of ECG under Physiological Constraints on the Solution Range
}

\author{
D Potyagaylo, WHW Schulze, 0 Dössel \\ Institute of Biomedical Engineering, Karlsruhe Institute of Technology, Karlsruhe, Germany, \\ danila.potyagaylo@ibt.uni-karlsruhe.de
}

\section{Introduction}

The inverse problem of ECG consists in recovering cardiac sources from body surface potential recordings. The problem is known to be ill-posed, i.e. small deviations in the data can lead to prominent changes in the solution and the solution itself is not unique. The most common approach for solving such problems is to use regularization, introducing penalty term and minimizing the weighted sum of data mismatch and penalty function.

\section{Methods}

Tikhonov regularization imposes penalty on the L2-norm of solution spatial derivatives. Although generally it performs well, the solution range is not taken into account. The interval for transmembrane potentials is known to be approximately from -90 to $+25 \mathrm{mV}$. Adding these constraints leads to an optimization formulation of the problem.

The L2-norm method causes considerable smoothing of the solution. Using L1-norm of penalty function instead of L2 and replacing the Laplace operator with the gradient is a widely accepted method in the image reconstruction community which allows to preserve solution features. Together with range constraints this problem was solved by means of conic optimization techniques.

\section{Results}

The standard Tikhonov solution was compared with that obtained from the new optimization set-up. Both methods, L2 and L1 regularization including solution range information, gave superior results in tems of correlation coefficients and relative errors. Regularization in L1 sense, as expected, provided better preservation of spatial potentail gradients.

\section{Conclusion}

The approaches considered in this work to include physiological information about transmembrane potential spatial distributions in solving the ECG inverse problem perform overall better than standard Tikhonov formulation, albeit they are computationally expensive. 